\title{
LCLS accelerator operation and measurement of electron beam parameters relevant for the x-ray beam
}

\author{
Henrik Loos \\ SLAC National Accelerator Laboratory, 2575 Sand Hill Road, Menlo Park, CA 94025, USA
}

\begin{abstract}
The Linac Coherent Light Source (LCLS) as the world's first hard x-ray free electron laser in the range of 250 $\mathrm{eV}$ to $10 \mathrm{keV}$ at its fundamental wavelength has been operated as a user facility since 2009 with six experimental stations and an increasing range of x-ray beam parameters now available to users. Various aspects of operating the LCLS accelerator to deliver the necessary electron beam in terms of bunch charge, energy, and length for the wide parameter range and different FEL operating modes will be discussed. An emphasis will be on the electron beam diagnostics that are most critical for generating the desired x-ray beam properties. Measurements of electron beam energy, energy loss, and transverse orbit will be shown as well as bunch duration and shape measurements.
\end{abstract}

Keywords: X-ray Free Electron Laser, Linear Accelerator, Electron Beam Diagnostics

\section{INTRODUCTION}

The Linac Coherent Light Source is one of two world-wide operating free electron laser (FEL) based hard and soft x-ray user facilities. ${ }^{1,2}$ It delivers photon pulses of several mJ pulse energy with durations of 10s to 100 s of femtoseconds at rates of up to $120 \mathrm{~Hz}$ to one of the six experiment hutches. The electron beam is generated with a high-brightness photo-injector, accelerated in three stages with two interspersed magnetic chicane bunch compressors, and finally delivered to the $130 \mathrm{~m}$ long undulator beam line where the x-ray beam is generated via self-amplified spontaneous emission from the electrons passing through the undulators. The main baseline and normal operation parameters for the LCLS electron and x-ray beam are summarized in Table 1.

Table 1. LCLS Parameters.

\begin{tabular}{|l|r|r|l|}
\hline & Baseline & Operation & Unit \\
\hline Repetition rate & 120 & $1-120$ & $\mathrm{~Hz}$ \\
\hline Electron energy & $4.3-13.64$ & $2.4-15.4$ & $\mathrm{GeV}$ \\
\hline Bunch charge & $200 \& 1000$ & $20-250$ & $\mathrm{pC}$ \\
\hline Bunch length & 20 & $<2-50$ & $\mu \mathrm{m}(\mathrm{rms})$ \\
\hline Emittance norm. & 1.2 & $0.13-0.5$ & $\mu \mathrm{m}$ \\
\hline X-ray energy & $0.83-8.3$ & $0.25-10.5$ & $\mathrm{keV}$ \\
\hline X-ray pulse energy & $<2$ & $<4.7$ & $\mathrm{~mJ}$ \\
\hline X-ray pulse length & 230 & $<5-500$ & fs (FWHM) \\
\hline
\end{tabular}

During the initial commissioning and following user operation, the operating range of LCLS and the capabilities to tailor the x-ray pulses in terms of wavelength, pulse duration, pulse energy, and spectral bandwidth have been continuously expanded with the latest addition of self-seeding for hard x-rays ${ }^{3}$ and a planned installation of a soft x-ray self seeding setup. User demands for this wide range of operating modes require frequent changes to

Further author information: (Send correspondence to H.L.)

H.L.: E-mail: loos@slac.stanford.edu, Telephone: 16509262324

Invited to SPIE Optics+ Optoelectronics Advances in X-ray Free-Electron Lasers II: Instrumentation Prague, Czech Republic April 15-18, 2013

Work supported by US Department of Energy contract DE-AC02-76SF00515 and BES. 


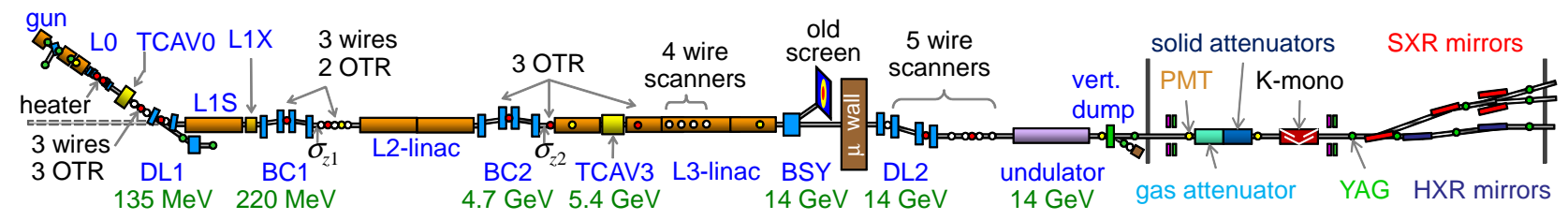

Figure 1. Schematic layout of the LCLS accelerator, undulators, and photon distribution lines with the locations and types of beam diagnostics indicated.

the accelerator setup, notably twice a day during the scheduled experiment destination switching, but also occasionally during a user shift itself. Automated accelerator setup and tuning procedures ${ }^{4}$ are important factors for an optimized photon beam delivery. Reliable diagnostics for the electron beam are similarly important, and the electron beam diagnostics relevant to the x-ray beam can be organized into two groups. One is the diagnostics needed for determining x-ray beam parameters from the electron beam, such as pulse length or pulse energy, which are not as easily accessible from the x-ray beam directly. The other relates to electron beam parameters that affect the x-ray parameters and that are important tools in understanding and optimizing the performance of the FEL. A schematic of the LCLS layout and the location of the most relevant diagnostics which will be discussed in the following sections are shown in Fig. 1.

\section{TRANSVERSE BEAM DIAGNOSTICS}

The FEL performance and the transverse properties of the generated x-ray beam depend strongly on the position and size of the electron beam as it passes through the $130 \mathrm{~m}$ of undulators at LCLS. The instrumentation used for measuring both parameters and the operational aspects are discussed in the following sections.

\subsection{Undulator Beam Position}

The main purpose of the beam position monitor (BPM) system in the undulators is to provide a precise measurement of the beam trajectory along the undulators at a level of at least a few $\mu \mathrm{m}$ resolution. This is then used by a feedback system to keep the beam orbit stable to a fraction of the beam size, and furthermore, to have high precision orbit information to perform the beam-based alignment of the undulators and focusing magnets so that an absolute straightness of the beam orbit over the $130 \mathrm{~m}$ long undulator system with deviations of less than $10 \mu \mathrm{m}$ is possible. The BPM system ${ }^{5}$ consists of 37 cavity BPMs with most of them located in the space between the undulators. Each has two cavities operating at X-band frequency, one a monopole cavity to provide beam intensity and normalization signals, and the other a dipole cavity to give the horizontal and vertical beam position. By correlating the shot by shot beam position at one BPM with the positions at all other BPMs, a resolution can be obtained which is about $250 \mathrm{~nm}$ at the upper end of the charge range of $250 \mathrm{pC}$, and $2 \mu \mathrm{m}$ at the lowest charge of $20 \mathrm{pC}^{6}$ This high resolution enables very precise alignment of the undulators to an absolute orbit straightness of better than a few $\mu \mathrm{m}^{7}$

\subsection{Undulator Beam Size}

The beam based alignment is done for a constant setting for the field strength of focusing magnets in the undulator over the entire energy range of the LCLS accelerator. This makes the beam transport through the undulators energy dependent and for each energy, the beam size and divergence at the undulator entrance has to be matched to the optimum beam optical functions to guarantee a more or less uniform beam size throughout the undulators. A so-called beam finder wire (BFW) system consisting of crossed $40 \mu \mathrm{m}$ thick carbon wires that can be inserted into the beam at the upstream end of each undulator ${ }^{7}$ was originally conceived to measure and align the position of this end of each undulator relative to the electron beam (the downstream end is adjusted by the adjacent BPM during beam based alignment). By stepping the horizontal of vertical position of the upstream undulator support girder, the respective wire can be moved through the electron beam, and the beam centroid and beam size can be obtained by simultaneously recording a beam loss signal as a function of wire position. Such a measurement at $5.2 \mathrm{GeV}$ is shown in Fig. 2 on the left panel. The signal scatter is mostly from 

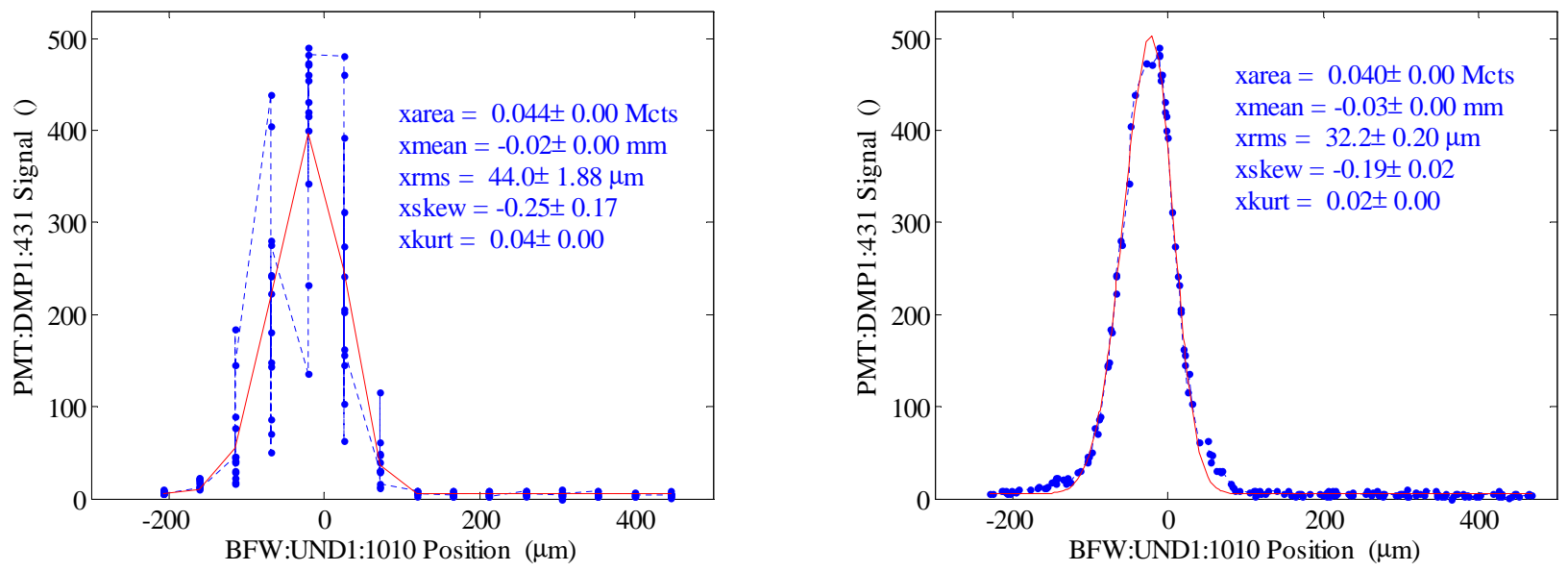

Figure 2. Beam loss signal at $5.2 \mathrm{GeV}$ from a single wire at undulator 10 vs. wire position (left panel) and corrected for beam jitter (right panel). The solid (red) lines are fits to an asymmetric Gaussian and the inserted text lists the different distribution moments from the fit lines.

beam orbit jitter, which does not affect the centroid position, but it increases the measured beam size by the jitter amplitude which can be a significant fraction of the beam size. A shot by shot measurement of the beam position from several adjacent BPMs and an orbit fit can be used to reconstruct the beam position at the wire for each shot. The right panel in Fig. 2 shows the same scan, but with the beam orbit-corrected wire position used instead. As a further improvement of the method the relatively slow movement of the support girder to move the wire position can be avoided altogether by introducing a deliberate orbit oscillation at the wire with suitable upstream correctors coils to sweep the beam across the wire instead. A complete beam profile measurement (not shown) can then be obtained within 5 seconds.

A measurement of the horizonal and vertical beam size with BFW scans at multiple locations along the

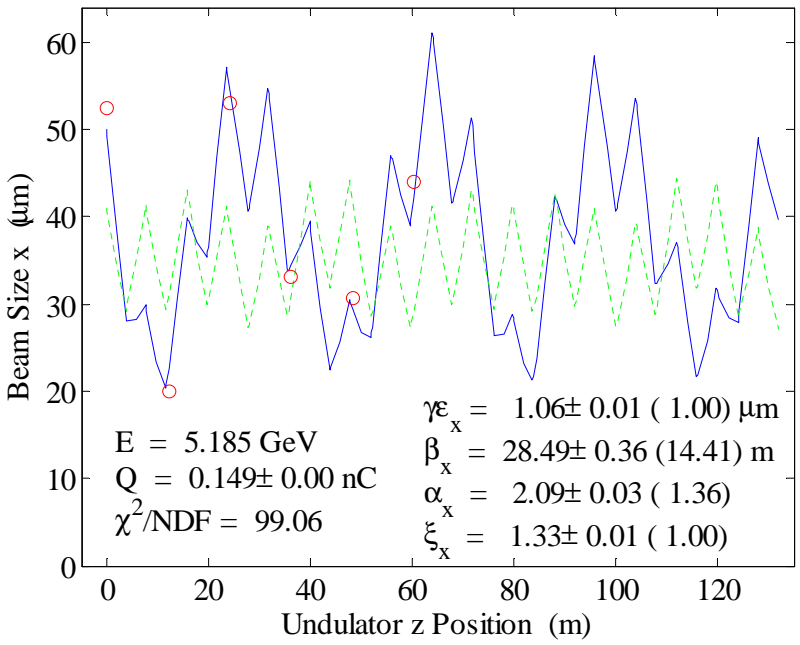

Figure 3. Beam emittance and matching in the undulators. The dots are the measured horizontal beam size at selected locations along the undulators, the solid line shows the evolution of the beam size from a fit to the data, whereas the dashed (green) line shows the ideal or matched beam size. The inserted text has the machine parameters and fitted Twiss parameters with $\xi$ being the so-called mismatch parameter. 
undulators can be used to fit the parameters of the transverse phase space ellipse at the beginning of the undulators and to determine the beam size evolution at any point throughout the entire undulator beam line. A horizontal beam size measurment at a beam energy of $5.185 \mathrm{GeV}$ with $6 \mathrm{BFWs}$ along the first half of the undulators is shown in Fig. 3 together with the predicted beam size based on the fit and the ideal ones. Both the measured $(\beta, \alpha)$ and ideal $\left(\beta_{0}, \alpha_{0}\right.$, in parentheses) values of the lattice functions are given for the $\mathrm{BFW}$ located $25 \mathrm{~m}$ into the undulators. The dimensionless mismatch parameter $\xi=\left(\beta \gamma_{0}-2 \alpha \alpha_{0}+\gamma \beta_{0}\right) / 2$ expresses the deviation from an ideal match, and for this particular example, the beam size though the undulators varied by a factor 3 instead of $30 \%$ in the matched case.

The established procedure for setting up the beam transport to the undulators is based on a measurement of the optical functions with standard LCLS wire scanners some $30 \mathrm{~m}$ and more upstream of the undulators. However, from past beam orbit measurements it is known that the beam transport in that area is not completely understood at beam energies much lower than the design energy of $13.6 \mathrm{GeV}$, which explains the observed mismatch. An undulator beam size-based matching procedure can now be used to fine tune the beam size evolution through the undulator, which in turn enables a better reproducibility and understanding of the x-ray divergence and beam size delivered to the x-ray experiment hutches.

\section{LONGITUDINAL BEAM DIAGNOSTICS}

Diagnostics based on the electron beam properties can provide valuable insight into the longitudinal electron phase space distribution which ultimately determine the spectral and temporal X-ray pulse properties. Measurements using existing instrumentation of the energy loss, energy spectrum, and temporal bunch length and distribution of the electron beam are discussed in the following sections. In addition, new bunch length and longitudinal diagnostics in the undulator area are presently being implemented to gain better knowledge of the bunch distribution close to the undulators as the magnetic bend systems in the long beam transport to the undulators can alter the temporal distribution in the electron bunches from the one measured after the second bunch compressor.

\subsection{Energy Loss Measurement}

The high resolution of a few $10 \mu \mathrm{m}$ of the BPM based transverse beam position measurement in the dispersive regions with $10 \mathrm{~cm}$ to $1 \mathrm{~m}$ dispersion enables a relative energy determination at a level of better than $10^{-4}$. The energy measurement can be further enhanced by removing beam orbit motion with a simultaneous beam position measurement at nearby BPMs. This can be utilized to measure the shot by shot difference in beam energy between dispersive sections upstream and downstream of the undulators to obtain the loss of electron beam energy due to the FEL interaction and hence the X-ray pulse energy. The additional energy loss from resistive wall wake fields of the beam pipe, from coherent synchrontron radiation in the bend magnets, and spontaneous radiation in the undulators can be accounted for with a reference measurement with the FEL interaction suppressed by creating a sufficiently large betatron oscillation of the beam in the undulators. The wake loss is furthermore dependent on the electron beam peak current and removing this correlation from the energy loss measurement significantly increases the signal to noise ratio in the data.

An energy loss scan at a beam energy of $8.22 \mathrm{GeV}$ where the first corrector in the undulators was used to introduce a betatron oscillation in the undulator with varying amplitude is shown in Fig. 4 with more than $20 \mathrm{MeV}$ loss per electron corresponding to about $6 \mathrm{~mJ}$ of X-ray pulse energy. The jitter of the electron beam peak current as measured with the relative bunch length monitor can be used to obtain its correlation (Fig. 4) to the wake loss, which is subsequently removed from the energy loss scan data. Such energy loss scans take only 10s of seconds and are performed regularly during normal LCLS operation, particulary when operating parameters are changed, to provide an accurate absolute calibration of the X-ray gas detectors, as their response depends strongly on the X-ray wavelength.

\subsection{Energy Spread Measurement}

The combination of off-crest acceleration and dipole chicanes used for the bunch compression in combination with longitudinal wake fields affecting the electron beam during the beam transport to the undulators generates a chirped electron energy distribution highly correlated with the bunch temporal distribution and highly sensitive 

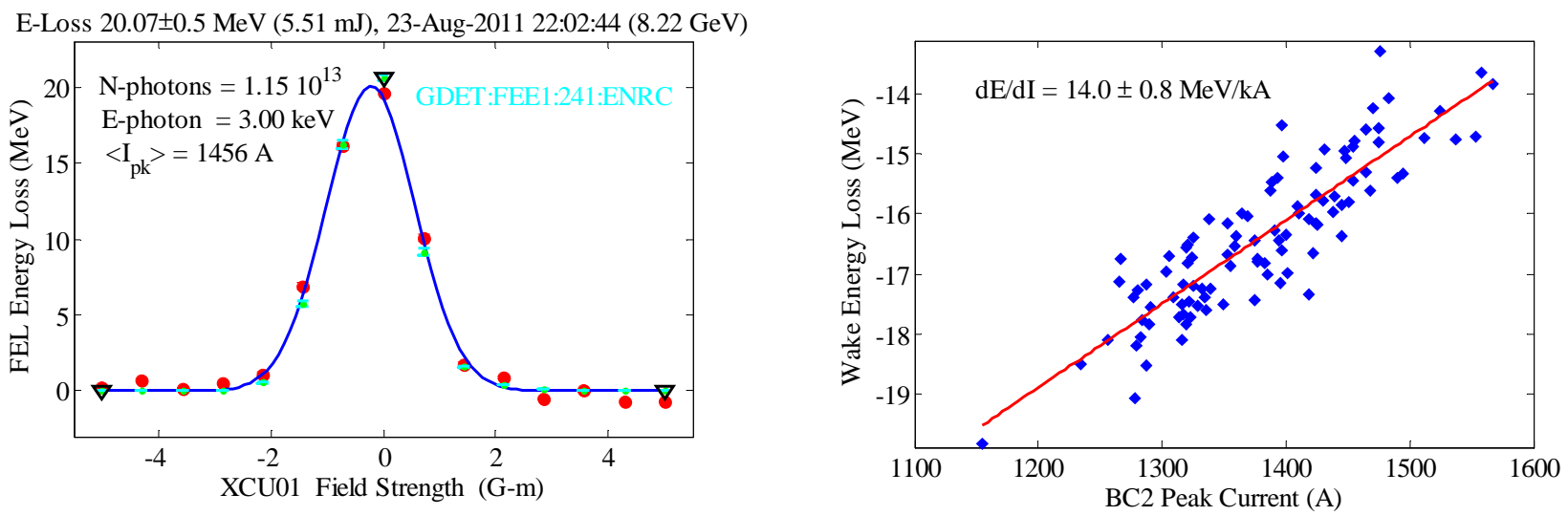

Figure 4. Electron beam energy loss measurement at $8.2 \mathrm{GeV}$ beam energy as a function of the field strength of the first corrector in the undulators (left panel). The red dots are the energy loss per electron, whereas the small green dots are corresponding X-ray pulse energy measurements using the X-ray gas detectors which were calibrated to match the electron beam measurement. The right panel shows a wake loss to peak current calibration (dots) with a corresponding linear fit (solid red line).

to the particular RF phase and amplitude settings for the accelerating sections. The energy spectrum of the X-ray beam is also mostly determined by the energy distribution in the electron bunch. High resolution measurements of the energy spread can be used to optimize the accelerator to achieve either a minimum X-ray bandwidth or one desired by the FEL users. Two wire scanners, one before and one after the undulators provide such measurements with a relative energy resolution at the level of $1 \times 10^{-4}$ and $3 \times 10^{-5}$. Similar to the above mentioned wire scans for the transverse beam distribution, simultaneous acquisitions of the beam orbit with fits to transverse beam position and, in this case, also beam energy are used to remove the effect of beam position and energy jitter on the scan data which can be as large as the energy spread itself.

Two scans under non-optimized RF settings before the first bunch compressor can be seen in Fig. 5 with the energy spectrum in the beam transport to the undulators in the left panel and the energy spectrum after the undulators in the main dump in the right panel. The beam orbit in the undulators was deliberately mis-steered
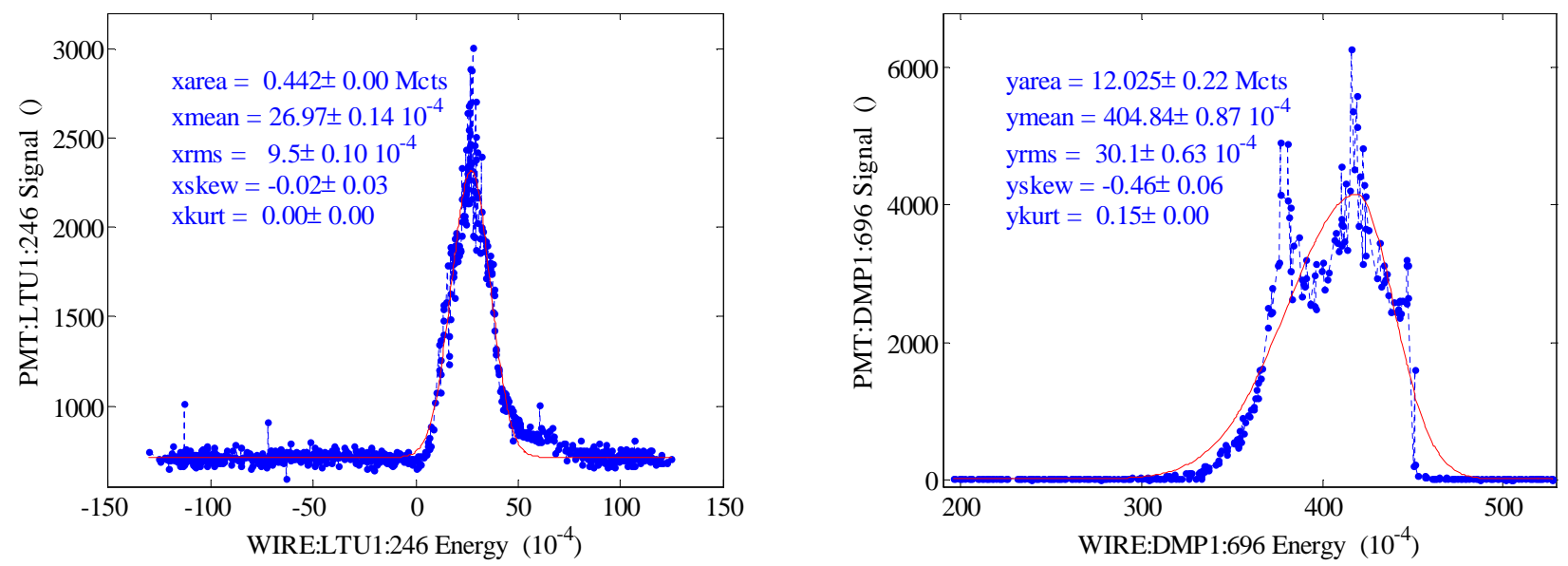

Figure 5. Measurements of electron beam energy spread under non-optimized beam conditions using two wire scanners in a dispersive region before (left panel) and after (right panel) the undulators. The dots represent the beam loss signal at each scan position corrected by beam motion and converted to relative beam energy, the solid (red) lines show asymmetric Gaussian fits to the data, and the text has the different distribution moments from the fit lines. 

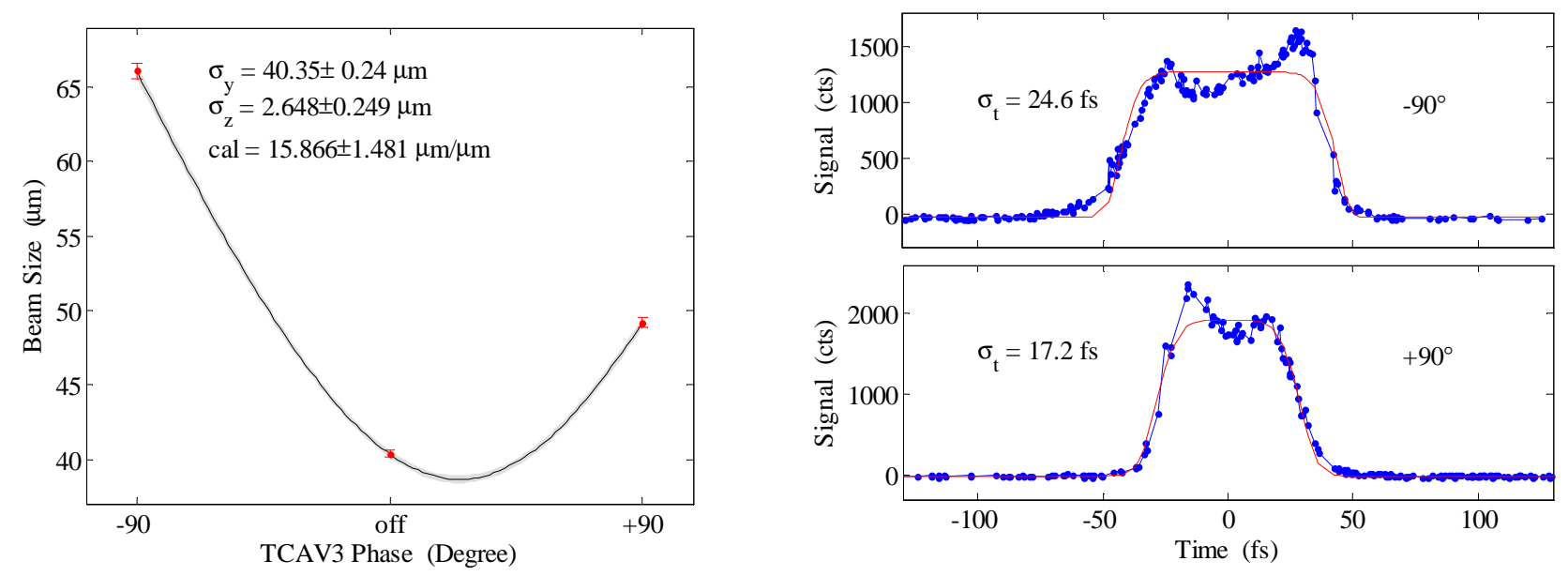

Figure 6. Bunch length and temporal profile measurements with deflecting cavity TCAV3. Left panel shows 3 measured beam sizes (red dots) at the $\pm 90^{\circ}$ phase and with the cavity off, and a fit of the rms bunch length to the data (solid line). The right panel shows for a longer bunch at a different bunch compression setting the two temporal distributions at the $\pm 90^{\circ}$ phases. The apparent difference in duration is due to a bunch tilt in the $(y, z)$-plane.

to suppress lasing and to remove the effect of the FEL interaction on the electron beam energy distribution. In this example the upstream relative energy spread of $10 \times 10^{-4}$ grows to $30 \times 10^{-4}$ after the undulator from resistive wall wake field effects upstream and within the undulators.

\subsection{Transverse Deflecting Cavity}

The absolute bunch length of the LCLS electron bunches after the second bunch compressor can be obtained with a so-called transverse deflecting cavity (TCAV3) ${ }^{8}$ whose RF fields are perpendicular to the beam direction to impart a horizontal or vertical time-dependent kick to the electron bunches. The amount of deflection is a function of the phase between the cavity and the beam, and if the beam travels through the structure at one of the zero-crossings of the field, the centroid of the bunch sees a zero kick, but the head and tail get deflected to opposing directions. This effectively maps the time coordinate to a transverse position if the beam profile is measured with a screen or wire scanner suitably located downstream with $90^{\circ}$ betatron phase advance. The $2.4 \mathrm{~m}$ long TCAV3 deflecting S-band cavity at $2856 \mathrm{GHz}^{9}$ is normally operated at a deflecting voltage of $15 \mathrm{MV}$ which results in a typical sheer factor of 10, i.e. the ratio of the vertical offset vs. longitudinal position. ${ }^{6}$ This factor is determined by changing the phase of the structure by a few degrees while observing the beam position at the wire scanner. At beam sizes between $40-70 \mu \mathrm{m}$, bunch structures of $20 \mathrm{fs}$ and $\mathrm{rms}$ bunch durations as low as $5 \mathrm{fs}$ can be resolved.

The transverse beam profiles streaked by the TCAV are measured with wire scanners some $300 \mathrm{~m}$ downstream of the cavity because due to coherent radiation issues no reliable beam profile could be obtained from the OTR screen originally intended for that purpose. The use of the wire implies a multi-shot measurement and the timing jitter between the RF fields in the cavity and the bunch leads at the wire to a large transverse jitter (comparable to the beam size) which has to be removed from the scan profile by a shot by shot orbit measurement with nearby BPMs.

A sample bunch length measurement close to the resolution limit is shown in Fig. 6 on the left panel for a $150 \mathrm{pC}$ bunch charge. A fit to the rms beam sizes (from Gaussian fits to the transverse profiles) at the $\pm 90^{\circ}$ zero-crossing phases and with the cavity off yields a rms bunch length of $2.5 \mu \mathrm{m}$ or $30 \mathrm{fs}$ FWHM assuming a flattop distribution. The shortest bunch lengths measured so far have been $1.5 \mu \mathrm{m}$ rms. Details of the longitudinal bunch structure which have great influence on the X-ray temporal shape are accessible from the streaked beam profile as shown in the right panel of Fig. 6 for an under-compressed bunch at normal operating conditions. The streaking at the two zero-crossing phases generates mirror images of the bunch distribution with the wake-field 
induced double-horn structure clearly visible. The significant difference between the durations at the two phase settings is indicative of a vertical tilt from transverse wake fields which is already present in the bunch upstream of the deflecting cavity.

\subsection{X-band TCAV Longitudinal Phase Space}

While sufficient in resolution for normal charge operation at $150-250 \mathrm{pC}$, the existing TCAV cavity cannot resolve the ultra-short sub-micron bunches generated at LCLS in the low charge mode at $20-40 \mathrm{pC} .{ }^{10}$ This diagnostic is also destructive to the beam. An X-band deflecting structure operating at $11.4 \mathrm{GHz}$ can achieve a much higher resolution from the increased frequency and the higher deflecting voltage. Two such $1 \mathrm{~m}$ long structures with a total deflecting voltage of $48 \mathrm{MV}^{11}$ are presently being installed and commissioned downstream of the LCLS undulators to provide a sheer factor of more than 100 which means a resolution of about 10 times higher as for the existing TCAV3. In combination with the magnetic bends in the main electron dump downstream of the new cavity it will be possible to observe the full longitudinal phase space on a Ce-doped YAG screen with the energy measured in vertical direction, and the temporal distribution in the horizontal direction from the transverse cavity deflecting the beam horizontally.

This measurement of the longitudinal phase space will provide a time resolved measure of the electron energy distribution along the bunch and - similarly to the aforementioned energy loss scan - can be used to compare the time resolved energy with and without FEL interaction to obtain the energy loss along the bunch, which in turn is closely related to the corresponding X-ray temporal distribution. Simulations of such measurements for both soft and hard X-rays show that a temporal resolution of $1-2 \mathrm{fs}$ can be achieved. ${ }^{12}$ The energy resolution with the present camera setting is about $5 \times 10^{-5}$. Planned upgrades to the existing YAG screen and camera system will provide a larger screen to accommodate the transverse jitter based on the cavity phase tolerance of $0.1^{\circ}$ and the beam arrival time jitter of about $50 \mathrm{fs}$. Furthermore, one can take advantage of the fact that this measurement is effectively non-destructive to the FEL as the X-band TCAV is located after the undulators, and with a faster image acquisition the reconstructed X-ray pulse profiles can be made available to the user experiment's data acquisition system at the full $120 \mathrm{~Hz}$ beam rate.

\subsection{Coherent Radiation Bunch Length Monitor}

The LCLS relative bunch length monitors ${ }^{13}$ are based on coherent edge radiation (CER) emitted from the last bend magnet in each bunch compressor chicane which is spectrally integrated by pyroelectric detectors within a certain bandwidth. The radiation is extracted from the vacuum beam pipe via an annular mirror to pass the electron beam through and hence provides a non-intercepting relative bunch length measurement used for the longitudinal feedback system. The output from the control system is a peak current value reported on a pulse by pulse basis assuming a flat-top temporal charge distribution related to the rms bunch length $\sigma$ and bunch charge $Q$ via

$$
I_{\text {Peak }}=\frac{Q c}{\sqrt{12} \sigma} .
$$

The calibration of the raw signal from the pyroelectric detectors is done by an absolute measurement of the rms bunch length with the transverse deflecting cavity over the normal operating range for bunch length and charge. A common for the emission of coherent radiation from an electron bunch, the radiation spectrum is a product of three contributions, the characteristic emission spectrum for edge radiation from a single particle, the Fourier transform of the temporal charge distribution, and the transmission and efficiency spectrum of the detection apparatus. A simplified analytical model of the detector signal as a function of bunch charge and length can be obtained if a Gaussian charge distribution is assumed, the CER single particle spectrum is taken as uniform and the transmission of the detection apparatus is modeled as a kind of bandpass filter accounting for low frequency diffraction losses with a power law of order $n$ dependency and the high frequency limit given by a Gaussian cut-off filter at frequency $\sigma_{f}$. With the charge distribution spectrum $I(\omega)$ and the spectral transmission $f(\omega)$ then given as

$$
I(\omega)=Q e^{-\omega^{2} \sigma^{2} / 2} \text { and } f(\omega)=\left(\frac{\omega}{\omega_{0}}\right)^{n} e^{-\omega^{2} \sigma_{f}^{2} / 2}
$$



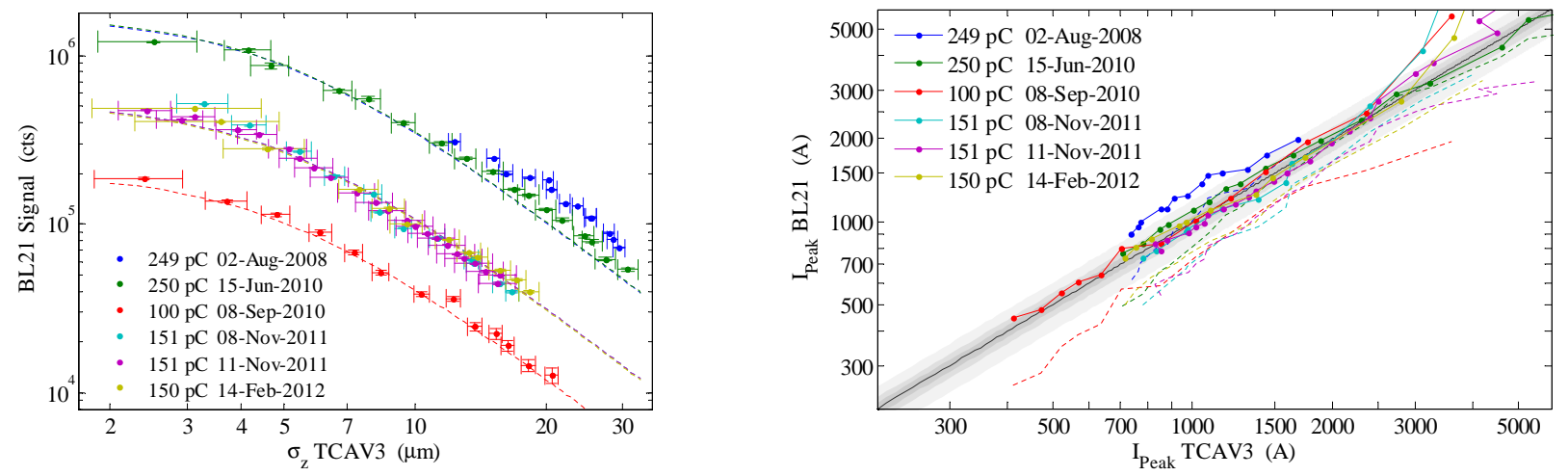

Figure 7. LCLS peak current calibration. Left panel shows raw digitizer signal for the BL21 bunch length monitor after $\mathrm{BC} 2$ vs. rms bunch length measured with transverse deflecting cavity TCAV3. The dashed lines are fits according to the modified Eq. (3). The right panel shows a comparison of new and old peak current calibration. The solid lines are based on a fit to the modified Eq. (3), whereas the dashed lines are based on a simple model with $n=1 / 6, \alpha=2$, and $\sigma_{f}=0$. The shaded areas indicate the $5 \%, 10 \%$, and $20 \%$ relative error levels.

the detected signal becomes proportional to

$$
S=\int|I(\omega) f(\omega)|^{2} \mathrm{~d} t=\mathrm{const} \frac{Q^{2}}{{\sqrt{\sigma^{2}+\sigma_{f}^{2}}}^{2 n+1}},
$$

with all constant factors lumped into one. As the bunch shape for the same rms bunch length is also depending on the bunch charge, the analytical model can further be enhanced by replacing the exponent of $Q$ in Eq. (3) with a variable exponent $\alpha$.

The calibration measurements taken over a 4 year operating period of the LCLS accelerator for the relative bunch length monitor (BL21) after the second bunch compressor (BC2) are shown in Fig. 7 compared to the absolute bunch length measurements with the transverse deflecting cavity (TCAV3) for bunch charges between $100 \mathrm{pC}$ and $250 \mathrm{pC}$ and $\mathrm{rms}$ bunch length ranges from $2 \mu \mathrm{m}$ to $30 \mu \mathrm{m}$. The fit lines for the different charge levels are based on a single set of parameters for Eq. (3) with $n=1 / 2, \alpha=2.35$, and $\sigma_{f}=5 \mu \mathrm{m}$. The best fit parameter for the filter cut-off parameter corresponds to a wavelength of about $27 \mu \mathrm{m}$ which matches well the cut-off wavelength of $30 \mu \mathrm{m}$ of the low-pass filter installed in front of the pyroelectric detector. Using Eqs. (1) and (3) the fit obtained above can be used to generate a calibration function to obtain the peak current from the bunch length monitor and compare it to the deflecting cavity data. This is shown in Fig. 7 on the right panel together with the presently used calibration based on a simple model with $n=1 / 6, \alpha=2$, and $\sigma_{f}=0$ or $S=$ const $Q^{2} / \sigma^{4 / 3}$. The new calibration better accounts for the signal saturation that occurs at the shortest bunch lengths when the bunch form factor becomes uniform within the detection spectral bandwidth. Most peak current values are within $5 \%$ of the ones measured with TCAV3, whereas the present calibration has up to $40 \%$ error and only agrees with the TCAV3 data at $250 \mathrm{pC}$ and between $1.5 \mathrm{kA}$ and $3 \mathrm{kA}$.

\subsection{Single Shot Spectrometer}

The relative bunch length monitor after the second bunch compressor does not provide spectral information to determine details of the temporal bunch distribution, and it cannot be calibrated properly for the ultra-short sub-10 fs long bunches in low charge operation as the absolute measurement with the nearby S-band TCAV does not provide enough temporal resolution. As a substantially less expensive alternative to an X-band TCAV, a single-shot near- to mid-infrared spectrometer can provide the bunch form factor and within certain limits the temporal distribution of the bunch using a suitable coherent radiation source like diffraction, edge, or transition radiation. Such a device (SSTPS or Single-Shot THz Prism Spectrometer) has been developed ${ }^{14}$ and is presently being benchmarked in the beam line section just upstream of the undulators where the coherent radiation is 
generated at an existing OTR foil. The spectrometer utilizes a KRS-5 prism as a dispersive element which is transmissive for the entire wavelength range of interest from $1-40 \mu \mathrm{m}$, and the dispersed radiation is then imaged onto a 128 element pyroelectric array. First tests of the spectrometer are giving promising results, and after commissioning of the X-band TCAV the bunch profiles reconstructed from the spectrometer measurements can be compared to the ones obtained from the TCAV.

\section{ACKNOWLEDGMENTS}

This work was supported by US DOE contract DE-AC02-76SF00515. Furthermore, I wish to thank all my colleagues at SLAC working on the LCLS accelerator for their invaluable contributions to the results discussed here.

\section{REFERENCES}

[1] Emma, P. et al., "First lasing and operation of an ångstrom-wavelength free-electron laser," Nature Photon. 4(9), 641-647 (2010).

[2] Ishikawa, T. et al., "A compact x-ray free-electron laser emitting in the sub-ångström region," Nature Photon. 6(8), 540-544 (2012).

[3] Amann, J. et al., "Demonstration of self-seeding in a hard-x-ray free-electron laser," Nature Photon. 6(10), 693-698 (2012).

[4] Loos, H., "Operational experience at LCLS," in [Proceedings of the 2011 FEL Conference, Shanghai, China], 166-172 (2010).

[5] Smith, S., Hoobler, S., Johnson, R. G., Straumann, T., Young, A., Lill, R. M., Morrison, L. H., Norum, E., Sereno, N., Waldschmidt, G. J., and Walters, D., "Commissioning and performance of LCLS cavity BPMs," in [Proceedings of the 2009 Particle Accelerator Conference, Vancouver, BC], 754-756 (2009).

[6] Loos, H. et al., "Operational performance of LCLS beam instrumentation," in [Proceedings of the 2010 Beam Instrumentation Workshop, Santa Fe, NM], 34-43 (2010).

[7] Nuhn, H.-D., "LCLS undulator commissioning, alignment, and performance," in [Proceedings of the 2009 FEL Conference, Liverpool, U.K.], 714-721 (2009).

[8] Altenmueller, O. H., Larsen, R. R., and Loew, G. A., "Investigations of traveling-wave separators for the Stanford Two-Mile linear accelerator," Rev. Sci. Instrum. 35(4), 438 (1964).

[9] Akre, R., Dowell, D., Emma, P., Frisch, J., Gilevich, S., Hays, G., Hering, P., Iverson, R., Limborg-Deprey, C., Loos, H., Miahnahri, A., Schmerge, J., Turner, J., Welch, J., White, W., and Wu, J., "Commissioning the Linac Coherent Light Source injector," Phys. Rev. ST Accel. Beams 11, 030703 (2008).

[10] Ding, Y., Brachmann, A., Decker, F.-J., Dowell, D., Emma, P., Frisch, J., Gilevich, S., Hays, G., Hering, P., Huang, Z., Iverson, R., Loos, H., Miahnahri, A., Nuhn, H.-D., Ratner, D., Turner, J., Welch, J., White, W., and Wu, J., "Measurements and simulations of ultralow emittance and ultrashort electron beams in the Linac Coherent Light Source," Phys. Rev. Lett. 102, 254801 (2009).

[11] Krejcik, P., Ding, Y., Frisch, J., Huang, Z., Loos, H., Wang, M.-H., Wang, J., Behrens, C., and Emma, P., "Design and fabrication of an X-band traveling wave deflection mode cavity for longitudinal characterization of ultra-short electron beam pulses," in [Proceedings of the 2012 Beam Instrumentation Workshop, Newport News, VA], THBP01 (2012).

[12] Ding, Y., Behrens, C., Emma, P., Frisch, J., Huang, Z., Loos, H., Krejcik, P., and Wang, M.-H., "Femtosecond x-ray pulse temporal characterization in free-electron lasers using a transverse deflector," Phys. Rev. ST Accel. Beams 14, 120701 (2011).

[13] Loos, H., Borden, T., Emma, P., Frisch, J., and Wu, J., "Relative bunch length monitor for the Linac Coherent Light Source (LCLS) using coherent edge radiation," in [Proceedings of the 2007 Particle Accelerator Conference, Albuquerque, NM], 4189-4191 (2007).

[14] Maxwell, T., Behrens, C., Ding, Y., Fisher, A., Frisch, J., and Loos, H., "Middle-infrared prism spectrometer for single-shot bunch length diagnostics at the LCLS," in [Proceedings of the 2012 International Beam Instrumentation Conference, Tsukuba, Japan], TUPA47 (2012). 